\title{
Combinatorial Tilings of the Sphere by Pentagons
}

\author{
Min Yan* \\ Department of Mathematics \\ Hong Kong University of Science and Technology \\ Kowloon, Hong Kong \\ mamyan@ust.hk
}

Submitted: Nov 16, 2012; Accepted: Mar 1, 2013; Published: Mar 8, 2013

Mathematics Subject Classifications: 05B45, 52C20

\begin{abstract}
A combinatorial tiling of the sphere is naturally given by an embedded graph. We study the case that each tile has exactly five edges, with the ultimate goal of classifying combinatorial tilings of the sphere by geometrically congruent pentagons. We show that the tiling cannot have only one vertex of degree $>3$. Moreover, we construct earth map tilings, which give classifications under the condition that vertices of degree $>3$ are at least of distance 4 apart, or under the condition that there are exactly two vertices of degree $>3$.
\end{abstract}

Keywords: combinatorial tiling; sphere tiling; pentagon

\section{Introduction}

Tilings are usually understood to be composed of geometrically congruent tiles. Typically we have finitely many prototiles and require that every tile to be isometric to one of the prototiles. For tilings of the plane by polygons, this means that there is a one-to-one correspondence between the edges of each tile and the edges of a prototile, such that the adjacency of edges is preserved, the edge length is preserved, and the angle between adjacent edges is preserved.

The combinatorial aspect of the tiling ignores the geometric information. For tilings of the plane, this means that we ignore the edge length and the angle information. What remains is only the number of edges of each tile. Dress, Delgado-Friedrichs, Huson [3, 4, $5,6]$ used the Delaney symbol to encode the combinatorial information and solved many combinatorial tiling problems. The Delaney symbol can also be turned into a computer

${ }^{*}$ Research was supported by Hong Kong RGC General Research Fund 605610 and 606311. 
algorithm for enumerating various classes of combinatorial tilings. For another research direction on the combinatorial aspects of tiling, see Schulte [10].

Our interest in the combinatorial tiling arises from the classification of edge-to-edge and monohedral tilings of the sphere. The tiles in such a tiling are all congruent and must be triangles, quadrilaterals, or pentagons. The classification of the triangular tilings of the sphere was started by Sommerville [11] in 1923 and completed by Ueno and Agaoka [12] in 2002. We are particularly interested in the pentagonal tilings, which we believe is relatively easier to study than the quadrilateral tilings because 5 is the "other extreme" among 3,4,5. In [7], we classified the tilings of the sphere by 12 congruent pentagons, where 12 is the minimal number of pentagonal tiles. Unlike the triangle case, where the congruence in terms of the edge length is equivalent to the congruence in terms of the angle, we needed to study different kinds of congruences separately, and then obtained the final classification by combining the classifications of different congruences. For 12 pentagonal tiles, the combinatorial structure is always the dodecahedron. Then we found all 8 families of edge congruent tilings for the dodecahedron. We also found 7 families of angle congruent tilings for the dodecahedron, plus perhaps around 20 families of angle congruent tilings for a remaining configuration of angles in the pentagon. This remaining case is yet to be completely classified, but is fortunately not needed for the final classification in [7].

The purpose of this paper is to study the possible combinatorial configurations beyond the minimal case of 12 pentagonal tiles. Of course this is only the first step in the complete classification. The next step is to separately study the edge congruence and the angle congruence. The final step is to combine the two congruences together. In [1], we further study edge congruent tilings, and completely classify for the case of the earth map tilings constructed in this paper. In [2], we further study geometrically congruent tilings, especially for the case of the earth map tilings. On the other hand, in $[8,9]$, we further study the numerics in angle congruent tilings.

Now we make precise our object of study. A tiling in this paper is naturally given by a graph embedded in the sphere, which divides the sphere into tiles that are homeomorphic to the disk. If the tiles are geometrically congruent pentagons, then the tiling has the following combinatorial property.

Definition. A combinatorial pentagonal tiling of the sphere is a graph embedded in the sphere, such that the boundary of any tile is a simple closed path consisting of fives edges, and the degree of any vertex is $\geqslant 3$.

A simple closed path of a combinatorial pentagonal tiling of the sphere divides the sphere into two disks. Each disk has their own combinatorial pentagonal tilings in the following sense.

Definition. A combinatorial pentagonal tiling of the 2-disk is a graph embedded in the disk, such that the boundary of the disk consists of some edges of the graph, the boundary of any tile is a simple closed path consisting of fives edges, and the degree of any vertex in the interior of the disk is $\geqslant 3$. 
The definition allows some vertices on the boundary to have degree 2 . If the boundary consists of $m$ edges, we also call the tiling a combinatorial pentagonal tiling of the $m$-gon.

Let $v_{i}$ be the number of vertices of degree $i$. Then it follows from the Euler equation and the Dehn-Sommerville equations that (see [7, page 750], for example)

$$
v_{3}=20+2 v_{4}+5 v_{5}+8 v_{6}+\cdots=20+\sum_{i \geqslant 4}(3 i-10) v_{i},
$$

and the number of tiles is

$$
f=12+2 \sum_{i \geqslant 4}(i-3) v_{i}
$$

In particular, the number of tiles must be even.

Our first result concerns the smallest number of tiles beyond the minimum 12 . In other words, is there a tiling of the sphere by 14 pentagons? Theorem 1 says that the tiling must have at least two vertices of degree $>3$, so that the next minimum number should be 16. In fact, we will see below that there is a unique combinatorial spherical tiling by 16 pentagons.

The equality above shows that vertices of degree 3 dominate the others. However, Theorems 4 and 5 say that vertices of degree $>3$ cannot be too isolated. In fact, any vertex of degree $>3$ has another vertex of degree $>3$ within distance 5 . Moreover, if there is a vertex $v$ of degree $>3$ such that there is no vertex of degree $>3$ within distance 3 of $v$, then the tiling must be the earth map tilings in Figures 11 and 19. In fact, vertices of degree $>3$ can be quite "crowded". For example, given any two combinatorial pentagonal tilings $T$ and $T^{\prime}$ of the sphere, we may construct the "connected sum" $T \# T^{\prime}$ by deleting one tile each from $T$ and $T^{\prime}$ and gluing the two together along the five boundary edges. The connected sum is a pentagonal tiling with all vertices along the five boundary edges having degree $>3$.

In general, we may define the earth map tilings to be the ones with exactly two vertices of degree $>3$. Naturally we call these two vertices poles. Theorem 6 says that for each distance between the poles ranging from 1 to 5 , there is exactly one family of earth map tilings. In increasing order of the distance, these families are given by Figures 23, 24, 25, 19 and 11.

Finally, we observe that the number of tiles in an earth map tiling is a multiple of 4 for distance 5 and a multiple of 12 for the other distances. Therefore the only combinatorial tiling by 16 pentagons is the earth map tiling of distance 5 .

\section{One Vertex of Degree $>3$}

Theorem 1. There is no combinatorial pentagonal tiling of the sphere with only one vertex of degree $>3$.

Let $P$ be a tile with the only vertex of degree $>3$ as one of its five vertices. Then the complement of $P$ is a combinatorial pentagonal tiling of pentagon, such that the only

THE ELECTRONiC JOURNAL OF COMBINATORICS 20(1) (2013), \#P54 
vertex of degree $>3$ is on the boundary. The following result implies that there is no such tiling.

Lemma 2. If a combinatorial pentagonal tiling of the m-gon, $m \leqslant 7$, has at most one vertex of degree $>3$, and all vertices in the interior have degree 3 , then the tiling either consists of only one tile, or is the complement of one tile in the dodecahedron tiling.

There is a very good reason why we stop at 7 . Figure 1 gives combinatorial pentagonal tilings of the 8-gon and the 9-gon. Their complements in the dodecahedron tiling are also combinatorial pentagonal tilings.
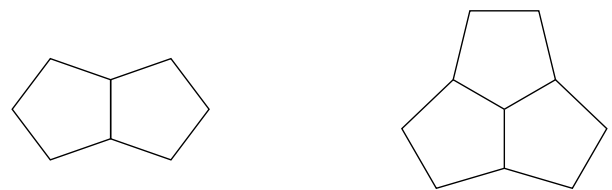

Figure 1: Pentagonal tilings of 8-gon and 9-gon.

A key technique we use for proving Lemma 2 and later results is the criterion for constructing pentagonal tiles. Let $e_{-}, e, e_{+}$be three successive edges, connecting $x, y, z, w$. We say that $e_{-}$and $e_{+}$are on the same side of $e$ if there is a path $\hat{e}$ connecting $x$ and $w$, such that $e_{-}, e, e_{+}, \hat{e}$ form a simple closed path, and the region enclosed by the simple closed path does not contain other edges at $y$ and $z$. In other words, all the edges at $y$ and $z$ other than $e_{-}, e, e_{+}$are outside of the region enclosed by the simple closed path. See left of Figure 2.
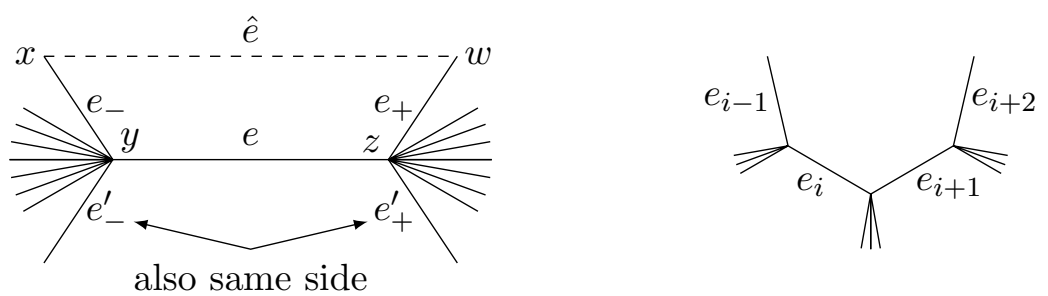

Figure 2: Edges on the same side.

Lemma 3. Suppose a sequence of edges $e_{1}, e_{2}, \ldots, e_{k}$ satisfies the following.

1. $e_{i}$ and $e_{i+1}$ share one vertex.

2. $e_{i-1}$ and $e_{i+1}$ are on the same side of $e_{i}$.

Then the edges in the sequence belong to the same tile. In particular, we have $k \leqslant 5$.

The conditions of the lemma are described on the right of Figure 2. In a combinatorial tiling of the sphere, any edge is shared by exactly two tiles. The tile on the side of $e_{i}$ that includes the corner between $e_{i-1}$ and $e_{i}$ is the same as the tile on the side of $e_{i+1}$ that includes the corner between $e_{i}$ and $e_{i+1}$. Lemma 3 follows from this observation. 
Proof of Lemma 2. Let us first restrict to $m \leqslant 5$, which is already sufficient for proving Theorem 1.

Under the given assumption, all the possible boundary configurations of the disk (with $m \leqslant 5)$ are listed in Figure 3. The only possible vertex of degree $>3$, which we will call the high degree vertex, has degree $n+2$ in the disk. Here "high" only means that there is no limit on how large $n$ can be, and the vertex can also have degree 2 or 3 on the other extreme. The lemma basically says that, with the exceptions of (5.1) with $n=0$ and (5.10) with $n=1$, all the other configurations will lead to contradiction.

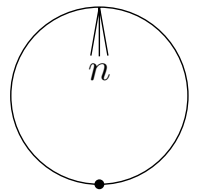

$(2.1)$

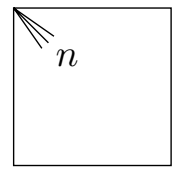

$(4.1)$

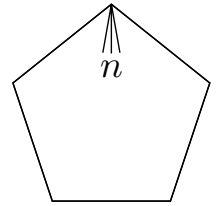

$(5.1)$

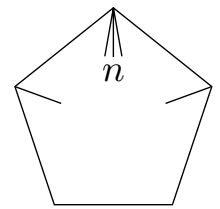

$(5.6)$

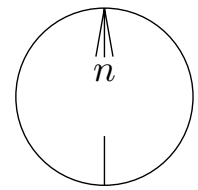

$(2.2)$

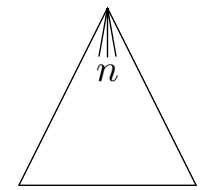

$(3.1)$

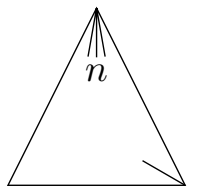

$(3.2)$

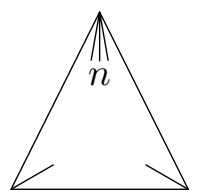

$(3.3)$
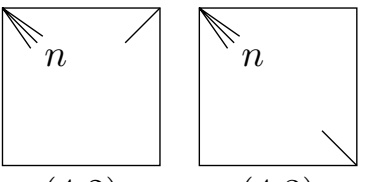

$(4.3)$

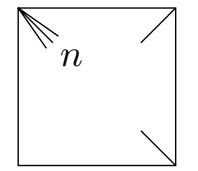

$(4.4)$

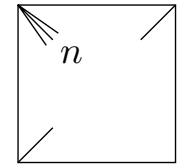

$(4.5)$

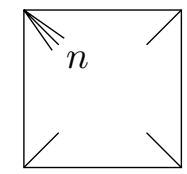

(4.6)

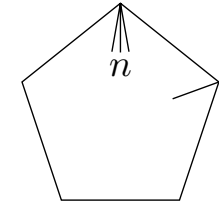

$(5.2)$

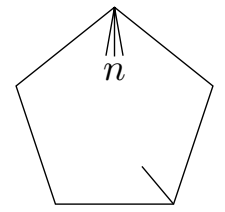

(5.3)

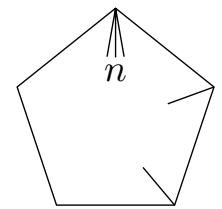

$(5.4)$

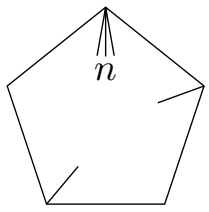

$(5.5)$

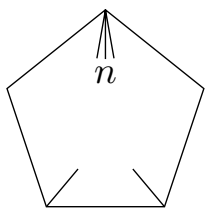

(5.7)

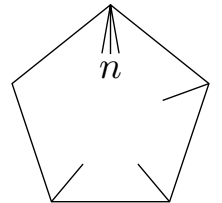

(5.8)

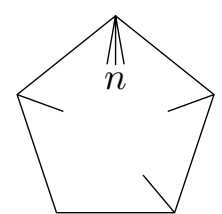

$(5.9)$

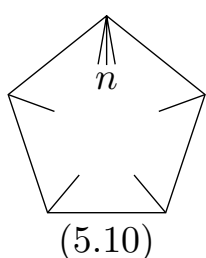

$(5.10)$

Figure 3: All boundary configurations, $m \leqslant 5$.

For the configuration (2.1), we apply Lemma 3 to the two boundary edges and find that they are the boundary edges of a pentagonal tile. However, all five boundary edges of this tile should form a simple closed path. Since two of the five edges already form a simple closed path, we get a contradiction. Similar contradictions can be obtained for (3.1) and (4.1).

For the remaining 18 configurations, if $n$ is sufficiently large, then we may try to build the tiles one by one by making use of the degree 3 assumption and Lemma 3 . When the remaining region becomes one of the configurations in Figure 3, we say that the original configuration is reduced to the new configuration.

Figures 4 and 7 give all the major (so called generic) reductions. There are also numerous non-generic reductions and some special reductions. The upshot is that the 
reductions allow us to prove by inducting on the number of tiles. The lemma is the consequence of the fact that, unless we start with $(5.1)_{n=0}$ or $(5.10)_{n=1}$, the reductions never conclude with $(5.1)_{n=0}$ or $(5.10)_{n=1}$. Therefore in the subsequent proof, an argument ends (or a case is dismissed) once we arrive at a configuration (sometimes one configuration among several) in Figure 3 that is neither $(5.1)_{n=0}$ nor $(5.10)_{n=1}$.

Figure 4 presents the generic reductions for the configurations (2.2), (3.2) and (3.3). The tiles are constructed with the help of Lemma 3, the degree 3 assumption, and the additional assumption (hence the name "generic") that any newly created vertex is not identified with an existing one unless it is absolutely necessary. We also note that, whenever an edge from the high degree vertex (i.e., the vertex of degree $n+2$ on the boundary) is used, we only use the edge closest to the boundary. This means that the angles $\alpha$ in Figure 4 do not contain any more edges.
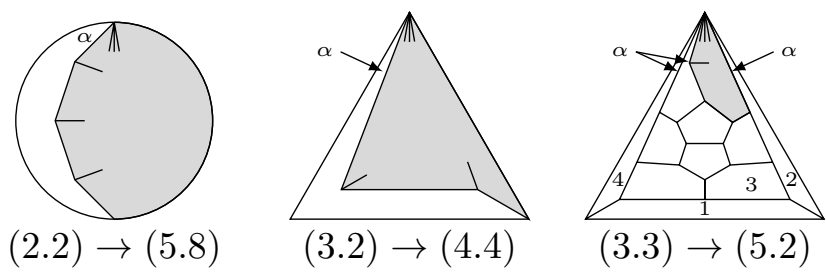

Figure 4: Generic reductions for two or three boundary edges.

Figure 5 shows many non-generic reductions for (3.3). Note that the non-generic reductions may conclude with more than one configurations. For example, the second reduction gives (2.1) and (4.4), and the third reduction gives (3.1) and (5.1) $)_{n=0}$. For the purpose of proving the lemma, we only need one of these not to be $(5.1)_{n=0}$ or $(5.10)_{n=1}$.

The reductions in Figure 5 are created as follows. First by Lemma 3, we may create the pentagonal tile 1 in Figure 4. This creates three new vertices. Since the five vertices of the tile 1 are distinct (because of the simple closed curve assumption), if there is any identification of a new vertex with the existing one, it has to be identified with the high degree vertex. Up to symmetry, the first two pictures in Figure 5 are all such identifications. Now we may assume the three new vertices are not identified with the existing ones and continue constructing the pentagonal tile 2 in Figure 4 . The two new vertices cannot be identified with the high degree vertex because the five vertices of the tile 2 must be distinct. When the two new vertices are identified with the existing vertices of degree 3, we get the third or the fourth picture. Then we may assume the two new vertices are "really new", and continue constructing the pentagonal tile 3 in Figure 4. The fifth and six pictures then indicate the cases that one of the vertices of the tile 3 is identified with the high degree vertex.

Analyzing each non-generic reduction at each step of the construction is rather tedious. An easy way to dismiss the non-generic reductions is to look at the distance between a new vertex and an existing one. For example, the gray region in Figure 6 is a newly constructed pentagonal tile, with two new vertices. The boundary of the remaining region of the triangle consists of 9 edges. Therefore if any of the new vertex is identified with the 


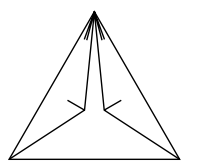

$\rightarrow(3.2) \quad \rightarrow(2.1)$

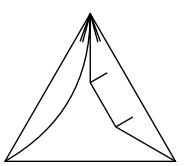

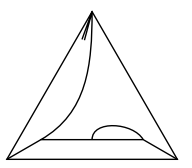

$\rightarrow(3.1)$

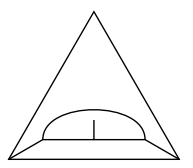

$\rightarrow(3.1)$

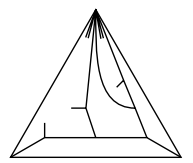

$\rightarrow(2.1)$

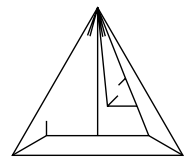

$\rightarrow(4.3)$

Figure 5: Other possible reductions of (3.3).

existing one, one of the regions we get will have no more than four edges on the boundary, which is of the form $(2 . *),(3 . *)$ or $(4 . *)$. Since this is neither $(5.1)_{n=0}$ nor $(5.10)_{n=1}$, such a reduction fits into our proof.

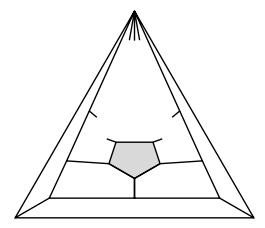

Figure 6: Easy way to dismiss non-generic reductions.

In general, if after constructing a new tile, the remaining region has no more than 10 boundary edges, then the identification of the new and existing vertices will lead to reductions. With this observation, we may easily dismiss all the non-generic reductions along the way and get the generic reductions in Figure 7 for the 4-gons and 5-gons. Moreover, by Lemma 3, the configuration (5.1) leads to a contradiction unless $n=0$, and the configuration (5.10) leads to a contradiction unless $n=1$.

Note that $n=0$ means the configuration actually becomes another simpler configuration. For example, we have $(2.2)_{n=0}=(2.1)_{n=1},(3.2)_{n=0}=(3.1)_{n=1},(4.5)_{n=0}=(4.4)_{n=0}$, and $(5.5)_{n=0}=(5.3)_{n=1}$. Therefore we may always assume $n \geqslant 1$, with $(5.1)_{n=0}$ as the only exception. On the other hand, in the following cases, we need bigger $n$ to carry out the generic reductions.

- $n \geqslant 2$ for $(4.6),(5.5),(5.9),(5.10)$.

- $n \geqslant 3$ for $(3.3)$, (5.8).

- $n \geqslant 4$ for $(5.7)$.

So it remains to reduce the special cases when the requirement on $n$ is not satisfied. These are listed in Figure 8. The only one that does not lead to contradiction is $(5.10)_{n=1}$, which is the complement of one tile in the dodecahedron tiling.

This completes the proof for $m \leqslant 5$. Now we turn to 6 -gons and 7 -gons. There are many possible configurations to consider. However, observe that if part of the boundary is as illustrated by the thick lines in Figure 9, then we can always produce the reduction. Moreover, we allow a vertex at the fringe of the thick lines to be the high degree vertex (see lower left corner of the first picture). 


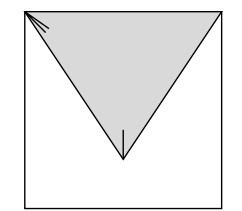

$(4.2) \rightarrow(3.2)$

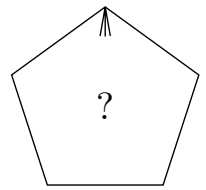

$(5.1) \rightarrow \times$

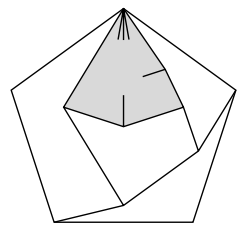

$(5.5) \rightarrow(5.5)$
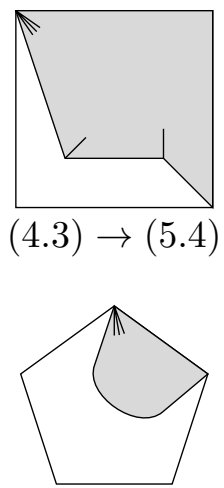

$(5.2) \rightarrow(2.1)$

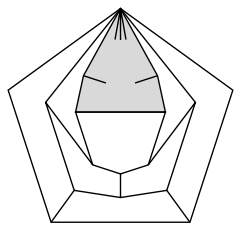

$(5.7) \rightarrow(5.6)$
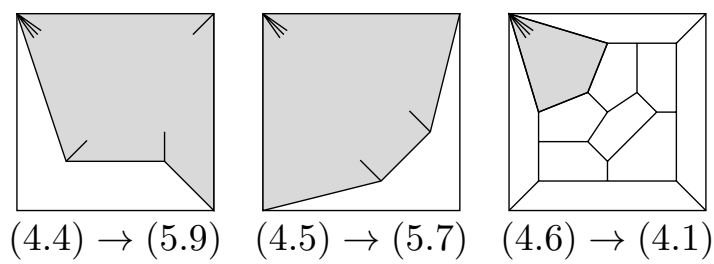

$(4.6) \rightarrow(4.1)$

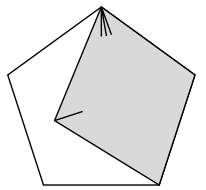

$(5.3) \rightarrow(4.2)$

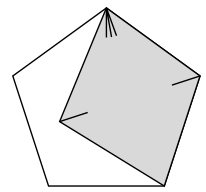

$(5.4) \rightarrow(4.5)$

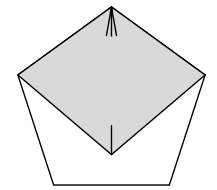

$(5.6) \rightarrow(4.3)$

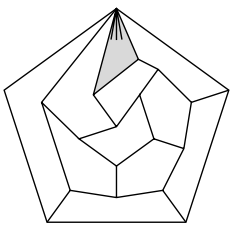

$(5.8) \rightarrow(3.1)$

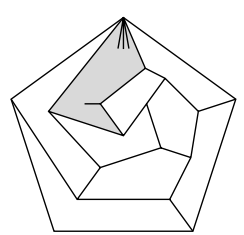

$(5.9) \rightarrow(5.3)$

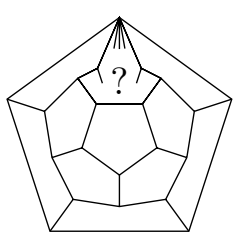

$(5.10) \rightarrow \times$

Figure 7: Generic reductions for four or five boundary edges.

So we only need to study those configurations not in Figure 9. It is also easy to see that the configurations with one high degree vertex and one or two vertices of degree 3 on the boundary either lead to contradictions or can be easily reduced. All the remaining non-trivial ones are illustrated in Figure 10.

We need to be concerned about the non-generic reductions. Since we have included 6gons and 7-gons in our study, a case can be dismissed if there are no more than 14 boundary edges. The observation is sufficient for dismissing all the non-generic reductions.

We also need to consider the special cases in which there are not enough edges at the high degree vertex for carrying out the reductions. In this regard, we only need to worry about the case $n=1$ for the fourth and the fifth in the second row in Figure 10. However, for $n=1$, they are respectively the second and the first in the second row. So the special cases have also been reduced.

Finally, we note that the reductions for the 6-gons and 7-gons never conclude with $(5.1)_{n=0}$ or $(5.10)_{n=1}$. Therefore all configurations eventually lead to contradictions.

\section{Distance Between Vertices of Degree $>3$}

Theorem 4. Suppose a combinatorial pentagonal tiling of the sphere has a vertex $\nu$ of degree $>3$, such that all vertices within distance 4 of $\nu$ have degree 3 . Then the tiling is the earth map tiling in Figure 11.

The tiling must have exactly two vertices $\nu$ and $\sigma$ (as the north and south poles) of degree $>3$, and the degrees at the two vertices must be the same. The first picture in 


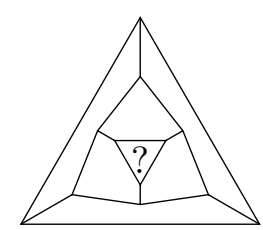

$(3.3)_{n=1}$

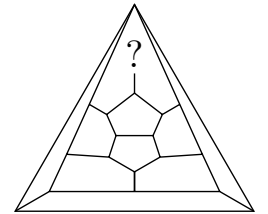

$(3.3)_{n=2}$

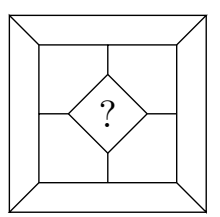

$(4.6)_{n=1}$

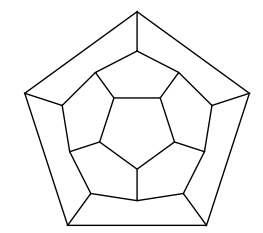

$(5.10)_{n=1}$

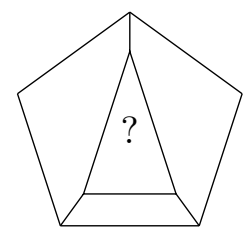

$(5.5)_{n=1}$

$(5.7)_{n=1}$

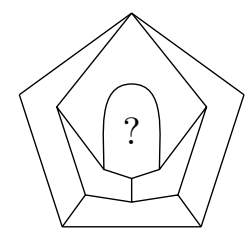

$(5.7)_{n=2}$

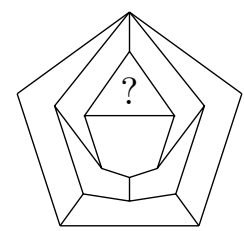

$(5.7)_{n=3}$

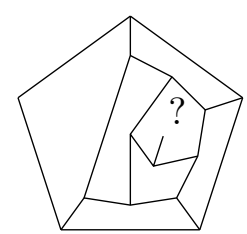

$(5.8)_{n=1}$

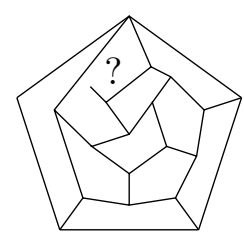

$(5.8)_{n=2}$

Figure 8: Special reductions.

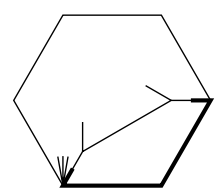

$(6) \rightarrow(7)$

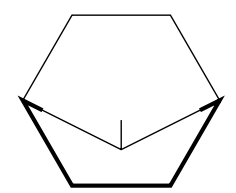

$(6) \rightarrow(5)$

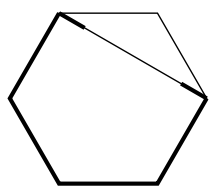

$(6) \rightarrow(3)$

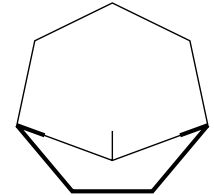

$(7) \rightarrow(6)$

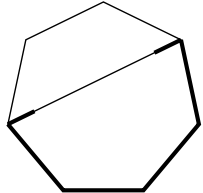

$(7) \rightarrow(4)$

Figure 9: Easy reductions for six or seven boundary edges.

Figure 11 is the view of the tiling from the north pole $\nu$. All the outward rays converge at the south pole $\sigma$. The second picture is our usual way of drawing maps, from the viewpoint of the equator.

An immediate consequence is that, if a combinatorial pentagonal tiling is not the earth map tiling in Figure 11, then every vertex of degree $>3$ must have another vertex of degree $>3$ within distance 4 .

Proof. Starting with a vertex $\nu$ of degree $>3$, we construct the pentagonal tiles layer by layer.

We apply Lemma 3 to get all the pentagonal tiles with $\nu$ as one vertex. This creates new vertices $x_{*}$ and $y_{*}$. Since the distance between $x_{*}, y_{*}$ and $\nu$ is $\leqslant 2<5$, the new vertices have degree 3 . We need to argue that all $x_{*}, y_{*}$ are distinct.

First, if $x_{i}=x_{j}$ for some $i \neq j$, then we get a 2-gon with $\nu$ and $x_{i}$ as the two vertices. Since $x_{i}$ has degree 3, the 2-gon is in one of the two cases in Figure 12. In the first case, by Lemma 3, the two boundary edges belong to the same tile. This contradicts to the requirement that all five edges of this tile should form a simple closed path. For the second case, we note that the outside is actually the first case. Therefore the second case also leads to a contradiction. This completes the proof that all $x_{i}$ are distinct.

Next, if all $x_{*}$ are distinct, and $x_{i}=y_{j}$, then we are in one of the three cases in Figure 13. In the first case, $\nu, x_{i}, x_{j}$ form a 3 -gon. We may apply Lemma 3 to this 3 -gon and 


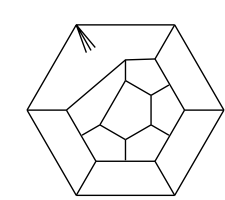

(6) $\rightarrow(5)$

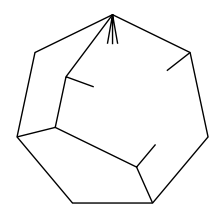

$(7) \rightarrow(7)$

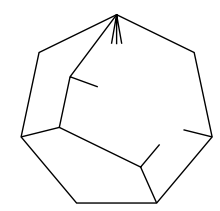

$(7) \rightarrow(7)$

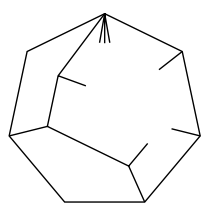

$(7) \rightarrow(7)$

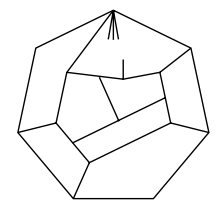

$(7) \rightarrow(6)$

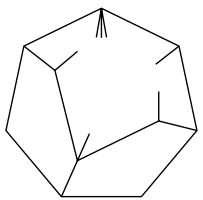

$(7) \rightarrow(7)$

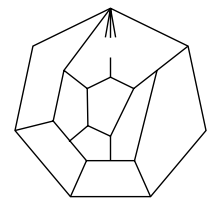

$(7) \rightarrow(7)$

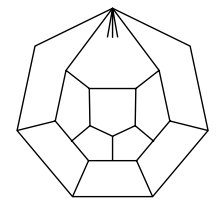

$(7) \rightarrow(5)$

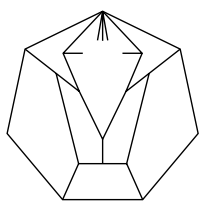

$(7) \rightarrow(6)$

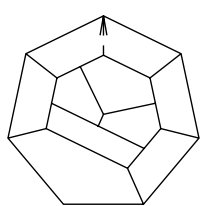

$(7) \rightarrow(7)$

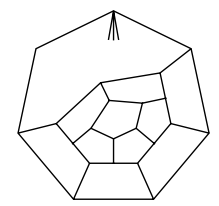

$(7) \rightarrow(7)$

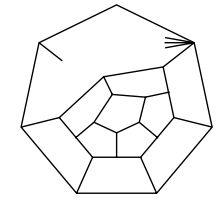

$(7) \rightarrow(7)$

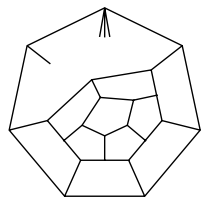

$(7) \rightarrow(7)$

Figure 10: Not so easy reductions for six or seven boundary edges.

find that the three boundary edges belong to the same tile. Like the first case of $x_{i}=x_{j}$, this contradicts to the requirement that the five boundary edges of the tile should form a simple closed path. For the second case, the outside is actually the first case, and we still get a contradiction. In the third case, we may apply Lemma 3 to the edges 1, 2, 3, 4 and get a pentagonal tile. The fifth edge 5 (the dashed line) means that we have $x_{i^{\prime}}=y_{j^{\prime}}$ for a pair $x_{i^{\prime}}, y_{j^{\prime}}$ closer than the pair $x_{i}, y_{j}$. So the argument can continue and eventually lead to a contradiction.

Finally, if all $x_{*}$ are distinct, $y_{*}$ are never identified with $x_{*}$, and $y_{i}=y_{j}$ for some $i \neq j$, then we are in one of the three cases in Figure 14. Note that we cannot have more than two $y_{*}$ identified together because the degree of the vertex would be $>3$.

Consider the first case. By applying Lemma 3 and the requirement that the five boundary edges of any pentagonal tile form a simple closed path, we see that the third edge at $y_{i}$ must point upwards. Moreover, without loss of generality, we may assume that those $y_{*}$ lying between $y_{i}$ and $y_{j}$ are no longer identified. So we get the black part of the first picture in Figure 15. Then we can apply Lemma 3 to develop the dashed part. Note that all the newly created vertices have distance $<5$ from $v$ and therefore have degree 3 . This implies that the new vertices are not identified with $y_{*}$ (i.e., all developments have to be generic). We see that the pattern repeats and therefore will lead to a contradiction.

Although the outside of the second case is the first case, the inside out trick that we used for proving $x_{i} \neq y_{j}$ cannot be used here, because in the proof of the first case of $x_{i} \neq y_{j}$, we used the additional assumption that those $y_{*}$ lying between $y_{i}$ and $y_{j}$ are no longer identified. By applying Lemma 3, we decide that the third edge at $y_{i}$ must point downwards. Like the proof of the first case, we may additionally assume that those $y_{*}$ lying between $y_{i}$ and $y_{j}$ are no longer identified. Then we can develop the tiling and get 

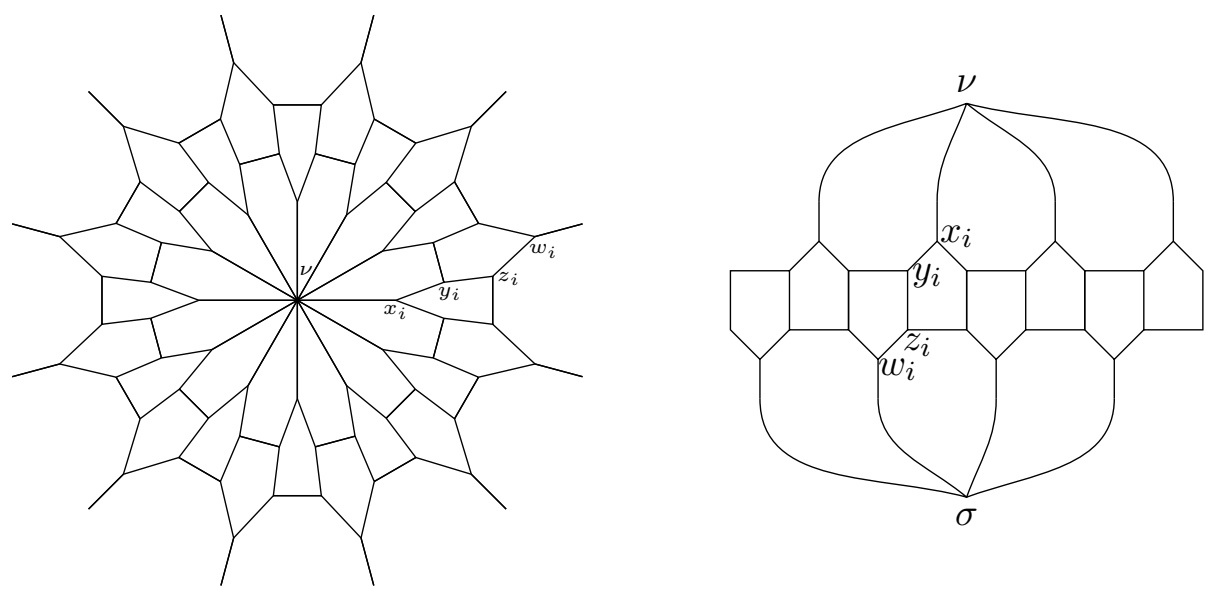

Figure 11: Earth map tiling, distance between poles $=5$.
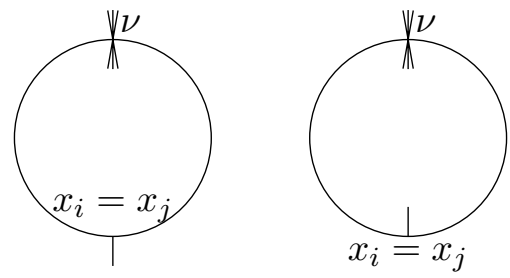

Figure 12: Two cases of $x_{i}=x_{j}$.

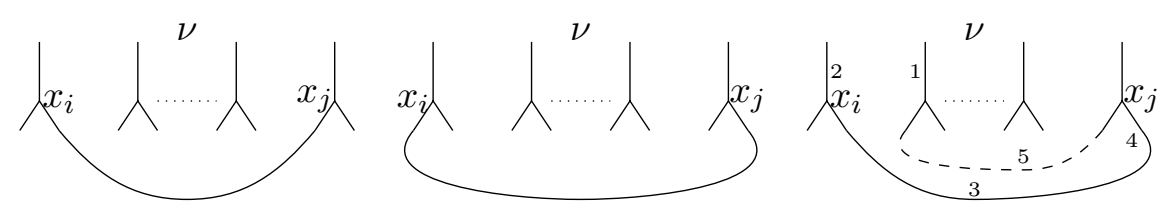

Figure 13: Three cases of $x_{i}=y_{j}$.
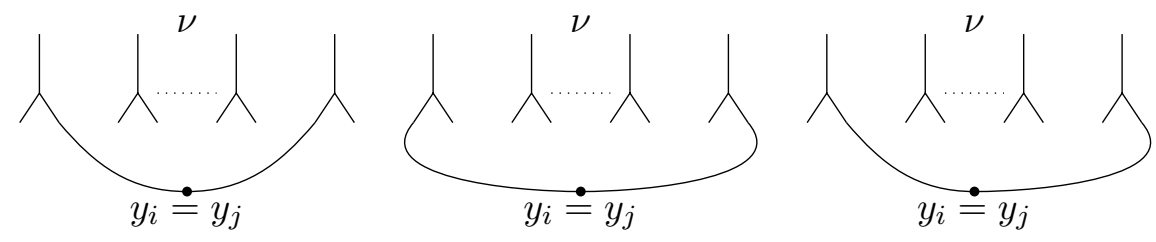

Figure 14: Three cases of $y_{i}=y_{j}$. 
the second picture in Figure 15. The picture is essentially the same as the first case and will lead to a contradiction.

Now we consider the third case, illustrated by the third picture in Figure 15. The dot represents the vertex $y_{i}=y_{j}$. If the third edge at $y_{i}$ points downwards, then applying Lemma 3 to the edges 1, 2, 3, 4 gives a pentagonal tile, with the fifth edge 7 as indicated in the picture. However, the edge 7 means that some $x_{*}$ and $y_{*}$ are identified, which has been proved to be impossible. On the other hand, if the third edge at $y_{i}$ points upwards, then applying Lemma 3 to the edges 3, 4, 5, 6 produces the edge 8, which also means some other $x_{*}$ and $y_{*}$ are identified. This proves that the third case is impossible.
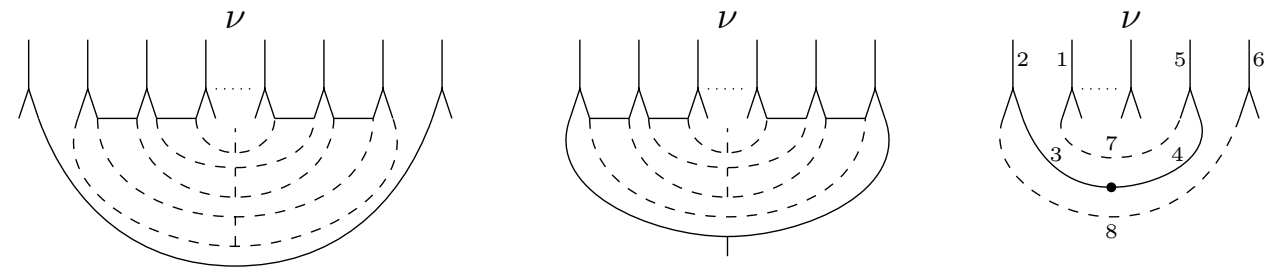

Figure 15: Impossible to have $y_{i}=y_{j}$.

After knowing all $x_{*}, y_{*}$ are distinct, we construct the second layer of pentagonal tiles and get new vertices $z_{*}, w_{*}$. Since the distance between $z_{*}, w_{*}$ and $\nu$ is $\leqslant 4<5$, the new vertices have degree 3 . We need to argue that all $x_{*}, y_{*}, z_{*}, w_{*}$ are distinct.

We know $x_{i}$ is connected to exactly three vertices $\nu, y_{i}, y_{i^{\prime}}$. If $x_{i}=z_{j}$, then the vertex $y_{j}$ connected to $z_{j}$ must be one of $\nu, y_{i}, y_{i^{\prime}}$. For topological reason, we cannot have $z_{j}=\nu$. So $j=i$ or $i^{\prime}$. But $z_{i}=x_{i}$ and $z_{i^{\prime}}=x_{i}$ contradict to the meaning of $z_{i}$ and $z_{i^{\prime}}$. This proves that $x_{*}, z_{*}$ are distinct.

If $y_{i}=z_{j}$, then we are in one of the three cases in Figure 16, indicated by the black part. Like the case of $y_{i}=y_{j}$, we may additionally assume that there is no more $y_{i^{\prime}}=z_{j^{\prime}}$ between the existing $y_{i}=z_{j}$. By successively applying Lemma 3 to the first case, we get the dashed part and conclude with the second case. Note that we created two new vertices in the process. If any of them is identified with $y_{*}$ or $x_{*}$, then we get either $x_{i^{\prime}}=z_{j^{\prime}}$, or $y_{i^{\prime}}=z_{j^{\prime}}$ between the existing $y_{i}=z_{j}$. Since these have been excluded, we get the generic development in the picture. For the second case, applying Lemma 3 gives a contradiction. For the third case, applying Lemma 3 again gives the dashed part, which is still the third case. Therefore we get another identification $y_{i^{\prime}}=z_{j^{\prime}}$ between the existing $y_{i}=z_{j}$, a contradiction.
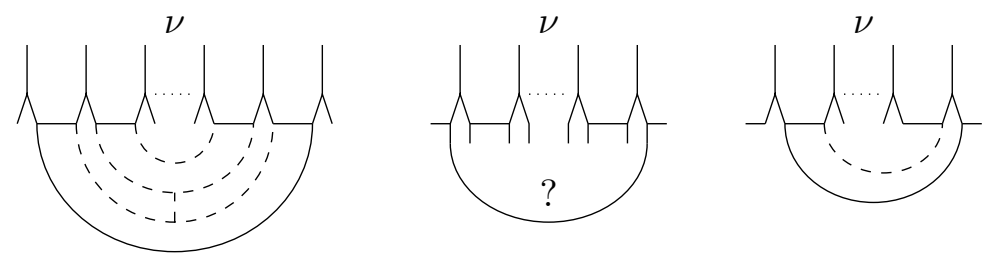

Figure 16: Three cases of $y_{i}=z_{j}$. 
If $z_{i}=z_{j}$ for some $i \neq j$, then we are in one of the three cases in Figure 17 . Note that we cannot have more than two $z_{*}$ identified together because the degree of the vertex would be $>3$. The outside of the first case is the second case. By Lemma 3, we conclude that the third edge at $z_{i}$ must point upwards in the second and third cases. Then we may further apply Lemma 3 to get the dashed part and the contradictions to Lemma 3.
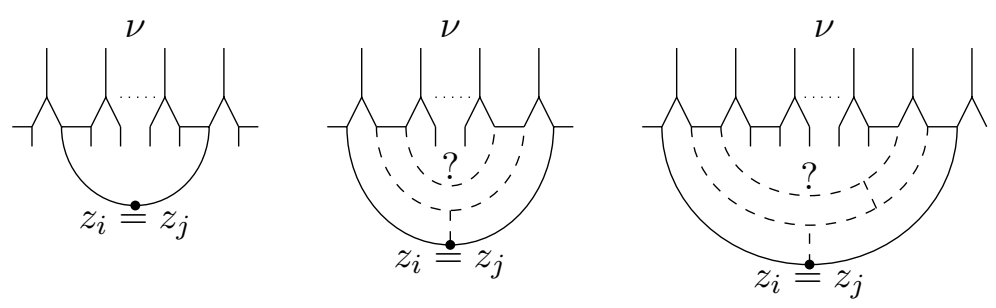

Figure 17: Three cases of $z_{i}=z_{j}$.

Now we know all $x_{*}, y_{*}, z_{*}$ are distinct. For topological reason, $w_{i}$ cannot be identified with $x_{*}$. Since $y_{*}$ have degree $3, w_{i}$ cannot be identified with $y_{*}$. Figure 18 shows all three cases for $w_{i}=z_{j}$. The outside of the first case is the second case. The second and third cases contradict Lemma 3 . Therefore $w_{i}$ cannot be identified with $z_{*}$. Finally, each $w_{i}$ is connected to two $z_{*}$. For $i \neq j, w_{i}$ and $w_{j}$ are connected to four distinct $z_{*}$. Since $w_{i}$ and $w_{j}$ have degree 3 , they cannot be identified.
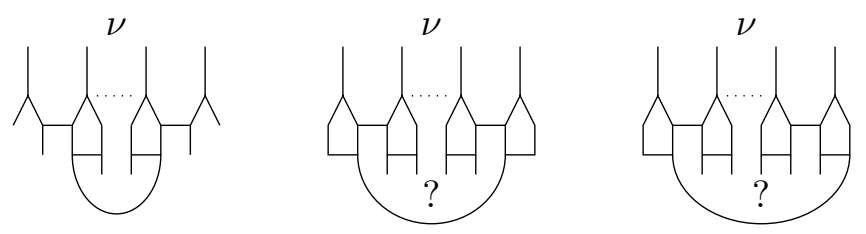

Figure 18: Three cases of $w_{i}=z_{j}$.

So we conclude that all $x_{*}, y_{*}, z_{*}, w_{*}$ are distinct. Then we may apply Lemma 3 to construct the next layer of tiles. By Lemma 3, we find that the third edges at $w_{*}$ converge at the same vertex. Therefore we get the earth map tiling in Figure 11.

Theorem 4 describes the case of maximal distance 5 between any vertex of degree $>3$ and the neighboring vertices of degree $>3$. The following is the next extreme case.

Theorem 5. Suppose a combinatorial pentagonal tiling of the sphere has a vertex $\nu$ of degree $>3$, such that all vertices within distance 3 of $\nu$ have degree 3 , and there is a vertex $\sigma$ of degree $>3$ at distance 4 . Then the tiling is the earth map tiling in Figure 19.

Proof. The proof of Theorem 4 is mostly based on no vertex of degree $>3$ within distance 3 of $\nu$. In fact, the only place we use no vertex of degree $>3$ within distance 4 is for proving $w_{*}$ are distinct. 

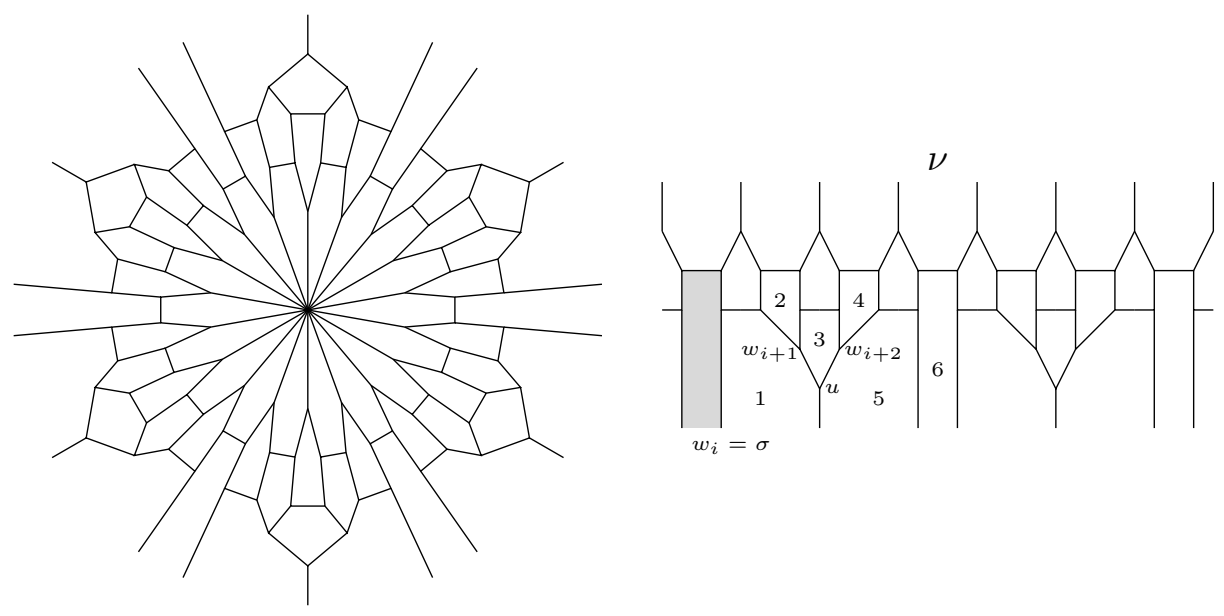

Figure 19: Earth map tiling, distance between poles $=4$.

So under the current assumption, $x_{*}, y_{*}, z_{*}$ are still distinct, and $w_{i}$ is not identified with any of $x_{*}, y_{*}, z_{*}$. Now we assume some $w_{i}$ has degree $>3$. On the right of Figure 19, the shaded region is one tile with $w_{i}$ as a vertex. We "open up" the tile at $w_{i}$ because $w_{i}$ will be the south pole $\sigma$. We assume that there are plenty of edges at $w_{i}=\sigma$.

We may construct the tile 1 by using Lemma 3 . The tile makes use of the next $w$ vertex $w_{i+1}$ and creates another new vertex $u$. Since the distance between $w_{i+1}, u$ and $\sigma$ is $\leqslant 2$, and the degree of $\sigma$ is $>3$, both $w_{i+1}$ and $u$ have degree 3 . Like the proof of Theorem 4 , this implies $w_{i+1}$ cannot be identified with any other $w_{*}$ (because otherwise $w_{i+1}$ would be connected to four distinct $z_{*}$ ). If $u=z_{j}$, then $w_{i+1}$ is identified with another $w_{*}$, a contradiction. If $u=w_{j}$, then by the degree 3 property of $w_{i+1}$ and $u$, we have the first picture in Figure 20, which contradicts Lemma 3. Therefore the construction of the tile 1 must be generic, as illustrated in Figure 19.
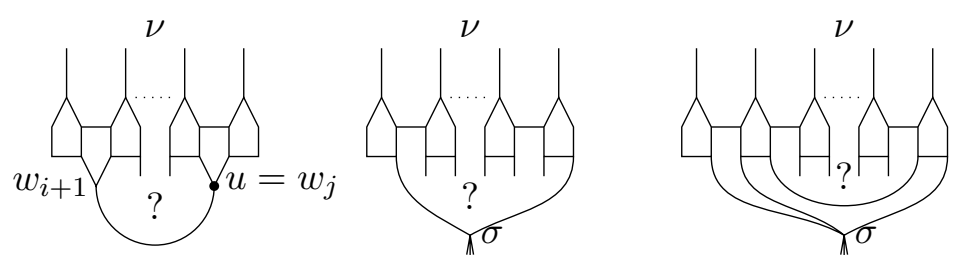

Figure 20: The new tiles are generic; and what happens when there are not enough available edges at $\sigma$.

After the tile 1, we immediately have the tiles 2 and 3 by Lemma 3 . Then the distance between $w_{i+2}$ and $\sigma$ is 2 . By the same reason as before, the degree of $w_{i+2}$ must be 3 , and the construction of the tiles must be generic. Then by Lemma 3 , we further get the tiles 4,5 and 6 .

Now the tile 6 is in the same configuration as the shaded tile we started with. Therefore the process repeats itself. The process stops when the edges at $\sigma$ are used up. This means that either we come back to the shaded tile in a perfect match, which gives the earth map 
tiling, or there is not enough available edges at $\sigma$ to complete the last "gap". The second and third pictures in Figure 20 show what happens when there are not enough available edges. Both lead to contradictions to Lemma 3.

\section{Two Vertices of Degree $>3$}

Theorems 4 and 5 cannot be further extended to smaller distances between vertices of degree $>3$. The connected sum construction in the introduction produces many vertices of degree $>3$ within distance 1 . Figure 21 (with four outward rays converging at the same vertex) is a combinatorial pentagonal tiling of the sphere with three vertices of degree 4 and eighteen tiles, such that the distance between any two vertices of degree 4 is 3 .

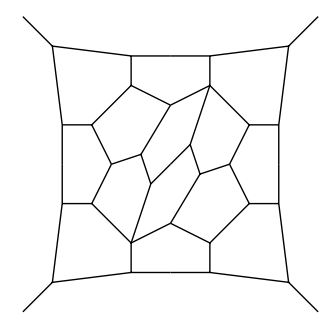

Figure 21: Three vertices of degree 4 and eighteen tiles.

In view of Theorem 1, therefore, we may study combinatorial pentagonal tilings of the sphere with exactly two vertices of degree $>3$. We expect to get similar earth map tilings with the two high degree vertices as the poles, even when the distance between the two poles is $\leqslant 3$. We call the shortest paths connecting the poles meridians. If the meridians have length 5 or 4, then the tilings have to be the earth map tilings in Figures 11 and 19. So we only need to consider meridians of length $\leqslant 3$. Up to the horizontal and vertical flippings, Figure 22 gives all the possibilities.

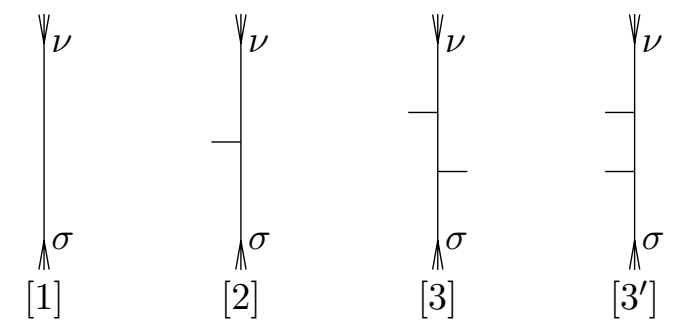

Figure 22: Possible meridians of length $\leqslant 3$.

We put any meridian in the vertical position and develop the tiling on the right side (or the east side). We find that the development comes back to the original meridian we started with. Therefore the same development can repeat itself. We call the tiling between adjacent meridians timezones. By glueing several timezones bounded by the same meridians, we get the earth map tilings. 
Figure 23 gives the earth map tiling with the meridian [1] (indicated by the thick line), in both the polar and equator views. Figure 24 gives the earth map tiling with the

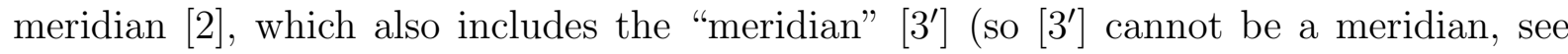
proof below). Figure 25 gives the earth map tiling with the meridian [3].
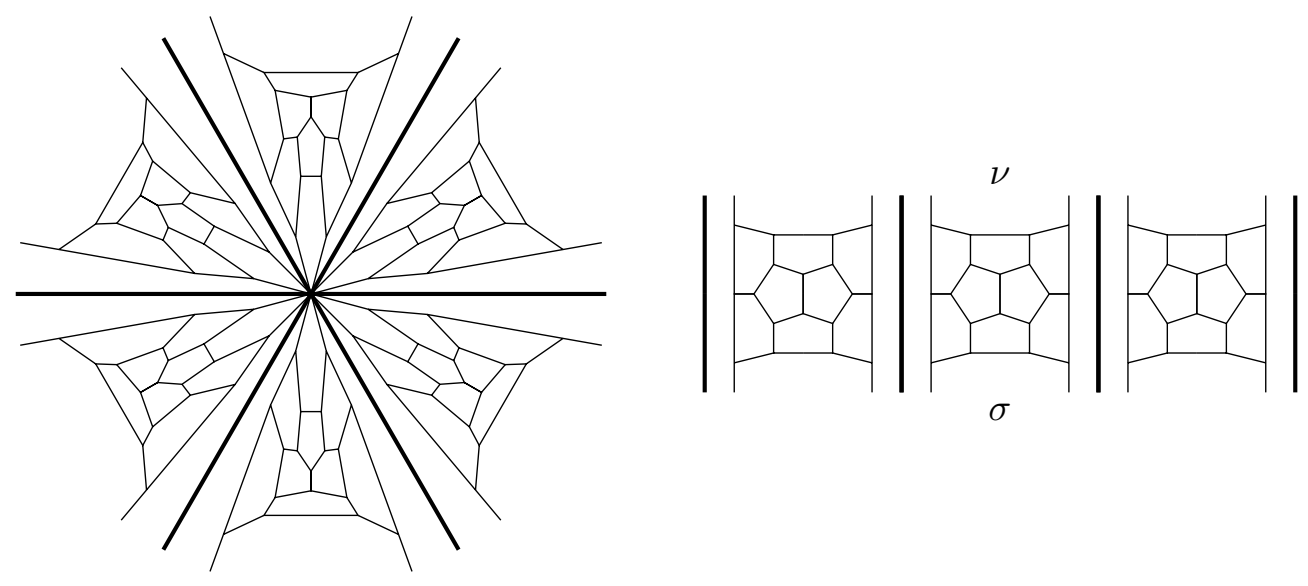

Figure 23: Earth map tiling, distance between poles $=1$.
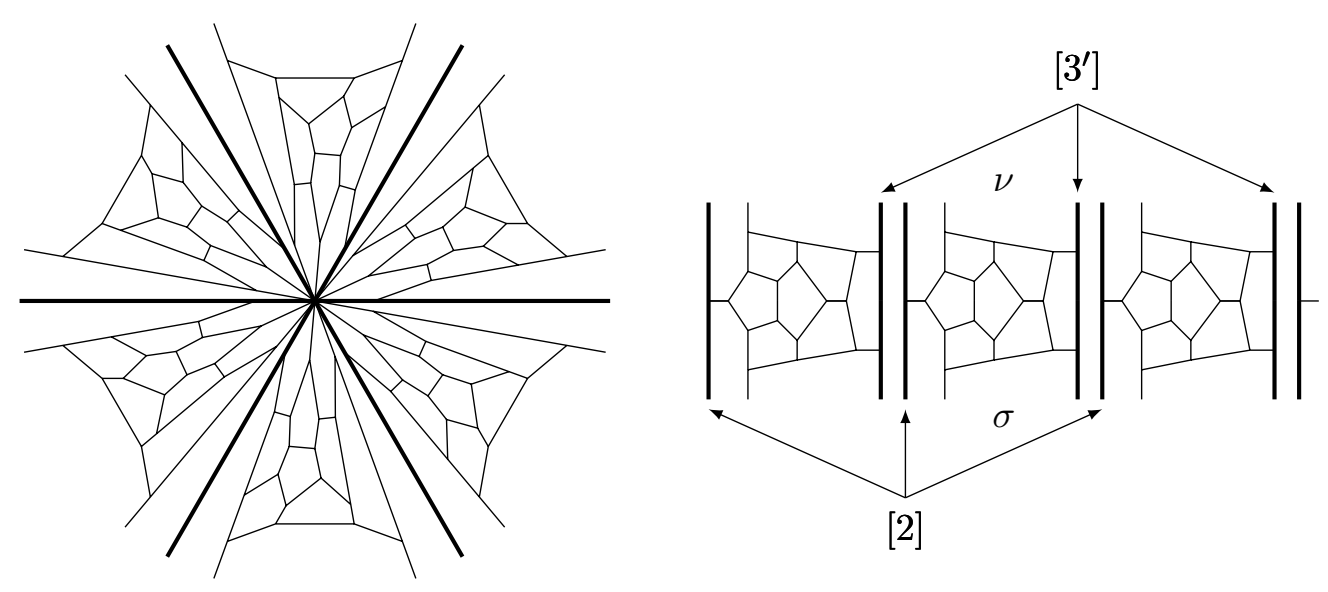

Figure 24: Earth map tiling, distance between poles $=2$.

Theorem 6. There are five families of combinatorial pentagonal tilings of the sphere with exactly two vertices of degree $>3$. The five families are given by Figures 11, 19, 23, 24, 25.

Proof. We need to argue three things. The first is what happens to the meridian [ $\left.3^{\prime}\right]$. The second is to justify the developments of the tilings in Figures 23, 24, 25 by dismissing the many possible non-generic cases. The third is to study the special cases that, after successively developing many timezones, there may not be enough available edges at the two poles to allow further development. 

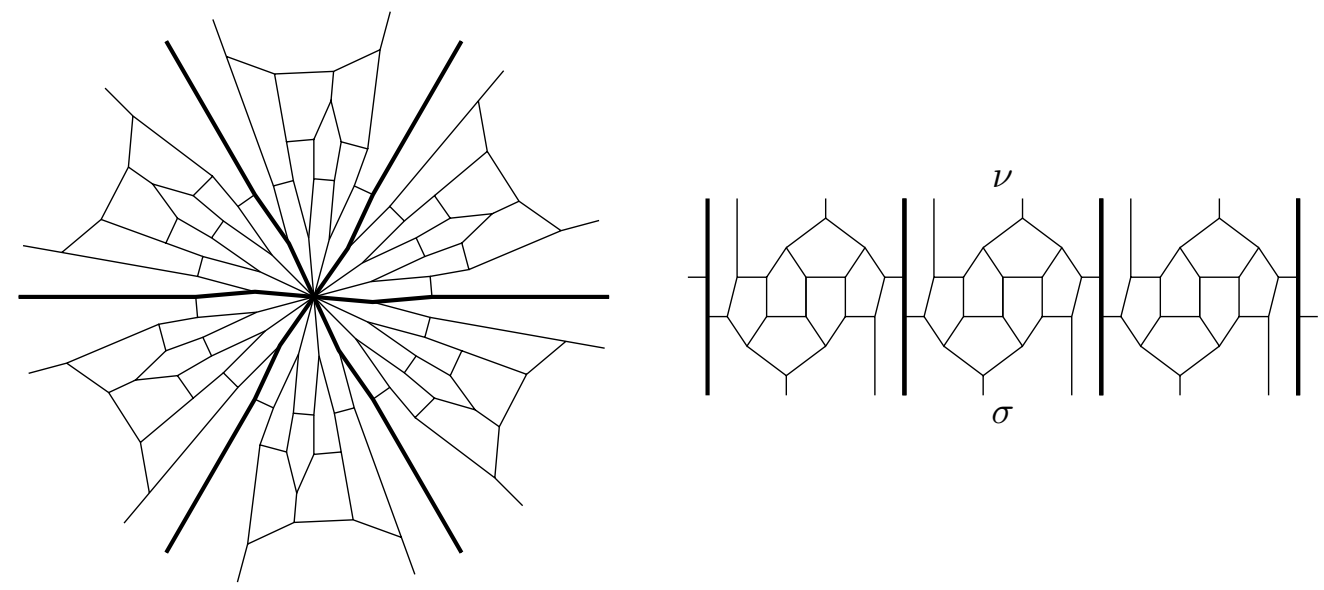

Figure 25: Earth map tiling, distance between poles $=3$.

First, the meridian $\left[3^{\prime}\right]$ is created at the end of developing the right side of the meridian [2]. If we start with $\left[3^{\prime}\right]$ and carry out the development, then we immediately get the meridian [2] on the right side and furthermore the same tiles in the equator view in Figure 24. So $[2]$ and $\left[3^{\prime}\right]$ give the same tiling. In particular, $\left[3^{\prime}\right]$ cannot be the shortest path and therefore cannot be the meridian.

Second, the non-generic developments can be dismissed for the reason largely similar to the proof of Lemma 2. The idea is that, after using Lemma 3 to create a new tile, the identification of a newly created vertex and an existing vertex produces a simple closed path. If the length of the path is $\leqslant 7$ and one of the regions divided by the path does not contain vertices of degree $>3$ in the interior, then Lemma 2 can be applied to the region to dismiss the case.

For example, we start with the meridian [3] and develop the tiles in Figure 26. In the first picture, the shaded tile is newly created, together with a new vertex indicated by the dot. If the dot is identified with the south pole $\sigma$, then we get the second picture, and we can apply Lemma 2 to the "obvious" simple closed path of length 6 .
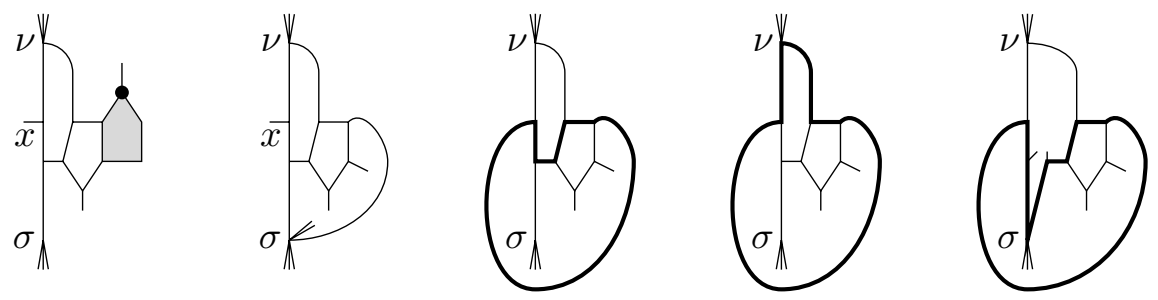

Figure 26: Non-generic developments starting from [3].

If the dot is identified with $x$, then we get the third picture. However, Lemma 2 can be applied neither to the inside nor to the outside of the thick simple closed path, because either side contains a vertex of degree $>3$ in the interior. To resolve the problem, we may either change the simple closed path to the one in the fourth picture and apply Lemma 
2 to the outside, or we may modify the simple closed path in the third picture to the one in the fifth picture and apply Lemma 2.

With suitable choice of the sequence of constructing tiles and extra care, we can indeed show that, starting from any meridian, the developments to the right side must be generic.
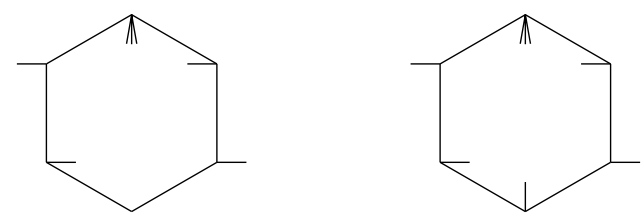

Figure 27: Failing cases for the meridian [3].

Now we deal with the third thing. We start with any meridian and add successive timezones. As long as we have at least two edges available at each pole, we may continue adding timezone. If there are exactly two edges available at each poles, then we can complete the final timezone and get the earth map tiling. This fails exactly when we have no or one edge left in one pole and at least one edge left at the other pole. Figure 27 describes the failing cases for the meridian [3]. Applying Lemma 2, these failing cases cannot happen. The same reason applies to the other meridians.

\section{Acknowledgements}

Part of the work is based on a HKUST UROP project by S. L. Wang and Y. Zhou. I would like to thank them for their contributions.

\section{References}

[1] K. Y. Cheuk, H. M. Cheung, M. Yan. Tilings of the sphere by edge congruent pentagons. arXiv:1301.0677, 2012.

[2] K. Y. Cheuk, H. M. Cheung, M. Yan. Tilings of the sphere by geometrically congruent pentagons for certain edge length combinations. preprint, 2013.

[3] O. Delgado-Friedrichs. Data structures and algorithms for tilings, I. Tilings of the plane. Theo. Comput. Sci., 303(2-3):431-445, 2003.

[4] O. Delgado-Friedrichs, A. Dress, D. Huson. Tilings and symbols: a report on the uses of symbolic calculation in tiling theory. Sém. Lothar. Combin., 34:Art.B34a, 1995.

[5] A. Dress. Regular polytopes and equivariant tessellations from a combinatorial point of view. In Algebraic topology, Göttingen 1984, volume 1172 of Lecture Notes in Math., pages 56-72. Springer, Berlin, 1985.

[6] A. Dress. Presentations of discrete groups, acting on simply connected manifolds, in terms of parametrized systems of Coxeter matrices-a systematic approach. Adv. in Math., 63(2):196-212, 1987. 
[7] H. H. Gao, N. Shi, M. Yan. Spherical tiling by 12 congruent pentagons. J. Combinatorial Theory Ser. A, 120(4):744-776, 2013.

[8] H. P. Luk. Angles in spherical pentagonal tilings. MPhil. Thesis, Hong Kong Univ. of Sci. and Tech., 2012.

[9] H. P. Luk, M. Yan. Angle combinations in spherical tilings by congruent pentagons. preprint, 2013.

[10] E. Schulte. Combinatorial space tiling. arXiv:1005.3836, 2010.

[11] D. M. Y. Sommerville. Division of space by congruent triangles and tetrahedra. Proc. Royal Soc. Edinburgh, 43:85-116, 1922-3.

[12] Y. Ueno, Y. Agaoka. Classification of tilings of the 2-dimensional sphere by congruent triangles. Hiroshima Math. J., 32(3):463-540, 2002.

\section{A Corrigendum - submitted 16th April 2014}

The proof of Theorem 4 contains an error. The edges labeled 3 and 4 on the right of Figure 13 are the same edge and should not be labeled separately. The picture is needed for showing that $x_{i}$ and $y_{j}$ are not identified. It turns out that showing $x_{i} \neq y_{j}$ also involves showing $y_{i} \neq y_{j}, y_{i} \neq z_{j}$ and $z_{i} \neq z_{j}$. We will prove all these non-identifications at the same time.

We will use the fact that the configurations in Figure 28 are impossible. This follows from Lemma 3 and the assumption (see the two definitions in the introduction) that all five boundary edges of any pentagonal tile form a simple closed path. Note that $n \geqslant 1$ for the pentagon in Figure 28, which means at least one edge pointing to the interior. The fact has been extensively used in the proof of Lemma 2 .
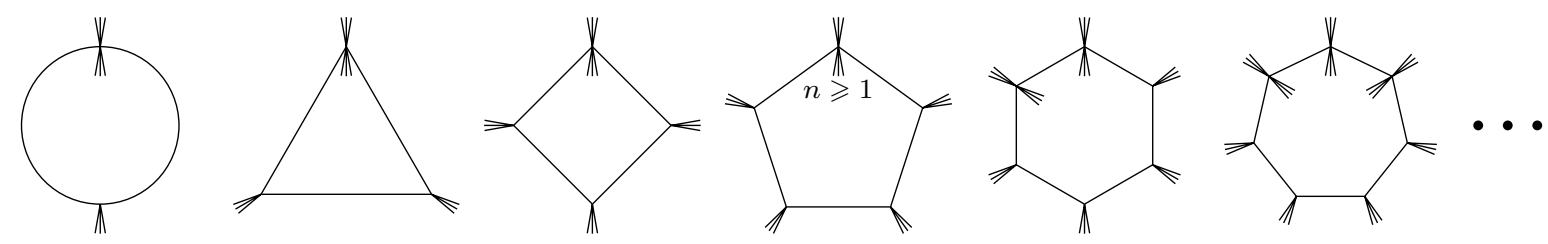

Figure 28: Impossible configurations.

The proof of Theorem 4 starts with a vertex $\nu$ of degree $k>3$. We label the edges emanating from $\nu$ by $k$ distinct indices $i$ and denote the other end of the $i$-th edge by $x_{i}$. Since $x_{*}$ are of distance 1 from $\nu$, they have degree 3 . If $x_{i}$ and $x_{j}$ are identified, then we get a 2-gon with $\nu$ and $x_{i}=x_{j}$ as the two vertices. Then either the inside or the outside of the 2-gon appears in Figure 28 (see original Figure 12) and is therefore impossible. This proves that all $x_{*}$ are distinct.

Each degree 3 vertex $x_{i}$ is connected to two vertices $y_{*}$ in addition to $\nu$. Since the distance between $y_{*}$ and $\nu$ are $\leqslant 2, y_{*}$ have degree 3 . If $x_{i}$ and $y_{j}$ are identified, then there are three possibilities, given in the first column of Figure 29. If two $y_{*}$ are identified, 
say $y_{i}=y_{j}$, then the identified vertex is a dot in the middle of the $x_{i} x_{j}$-edges in the first column, and we get the second column. Moreover, the third edge at the degree 3 vertex $y_{i}$ may point either upward or downward, so that we actually have six possibilities in total.
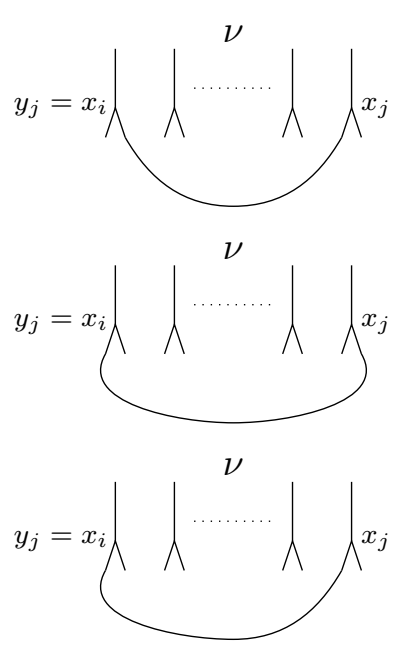
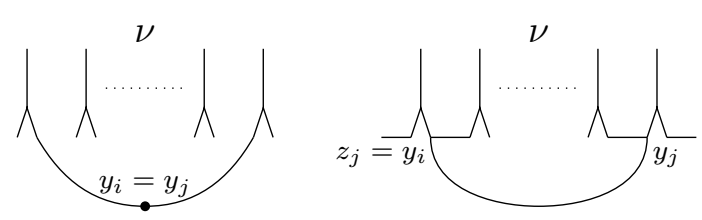

$\nu$
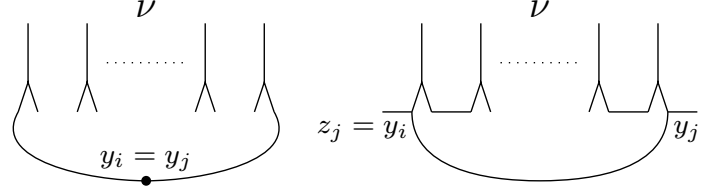

$\nu$

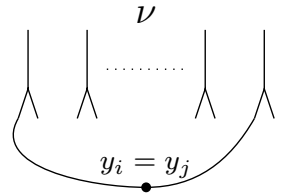

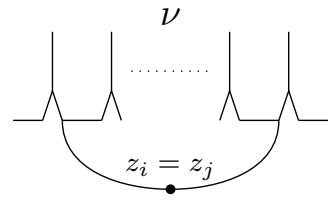

V

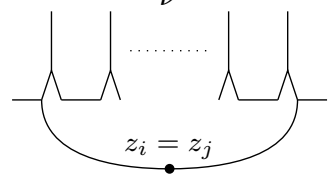

V

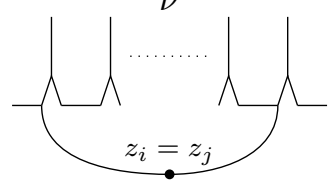

Figure 29: Identifications among $x_{*}, y_{*}, z_{*}$.

If all $x_{*}, y_{*}$ are distinct, then we may apply Lemma 3 to construct all the tiles at the vertex $\nu$, each having $\nu$, two $x_{*}$ and two $y_{*}$ as the five vertices. Now each degree 3 vertex $y_{*}$ is connected to one $x_{*}$, another $y_{*}$, and a new vertex $z_{*}$. Since the distance between $z_{*}$ and $\nu$ are $\leqslant 3, z_{*}$ have degree 3 . If $y_{i}$ and $z_{j}$ are identified, then we get three possibilities in the third column of Figure 29. If two $z_{*}$ are identified, say $z_{i}=z_{j}$, then the identified vertex is a dot in the middle of the $y_{i} y_{j}$-edges in the third column, and we get the fourth column. Moreover, considering the upward or downward direction of the third edge at the dot, we have six possibilities in total.

We claim that all the configurations in Figure 29 are impossible, so that all $x_{*}, y_{*}, z_{*}$ are distinct. We redraw the configurations as Figure 30. For example, the configurations in the first row of Figure 29 are respectively labeled (3.1), (4.1), (4.2), (5.1), (6.1), (6.2). The vertex $\nu$ is indicated by $n$ interior edges at the high degree boundary vertex.

The argument for the impossibility is based on the following facts about the configurations in Figure 30: Any configuration is part of a combinatorial spherical tiling. The boundary has a unique high degree vertex. Although the interior allows high degree vertices, any vertex of distance $\leqslant 3$ from the high degree boundary vertex has degree 3 . Note that although the theorem assumes degree 3 within distance 4 of the high degree boundary vertex, the stronger assumption of within distance 3 is need for proving Theorem 5 .

By Figure 28, (3.1) and (4.1) are impossible. By considering the outside of the configurations, (3.2) and (4.4) are also impossible. Moreover, applying Lemma 3 to (6.3) gives (3.1), which we have shown is impossible. Applying the lemma to the outside of (6.2) gives similar contradiction. We conclude that the configurations (3.1), (3.2), (4.1), (4.4), (6.2), (6.3) are all impossible. For the remaining configurations, we follow the argument in the proof of Lemma 2. All the non-generic reductions can be dismissed as before, so that we 


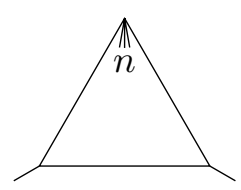

$(3.1)$

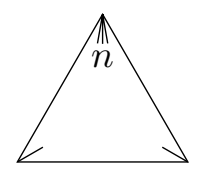

(3.2)

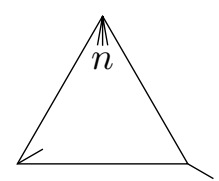

(3.3)

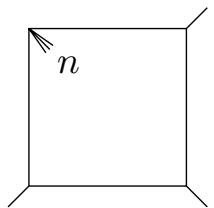

(4.1)

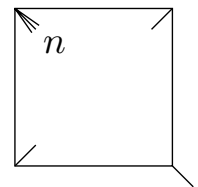

(4.3)

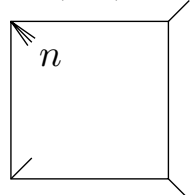

(4.5)

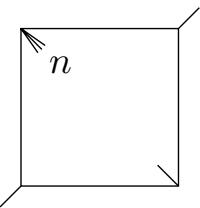

(4.2)

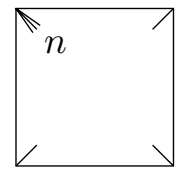

(4.4)

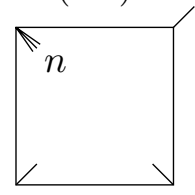

(4.6)

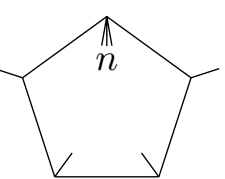

$(5.1)$

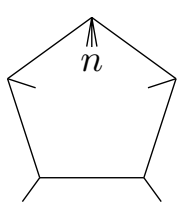

(5.2)

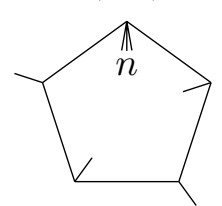

(5.3)

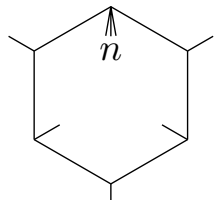

(6.1)

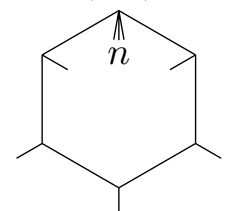

(6.3)

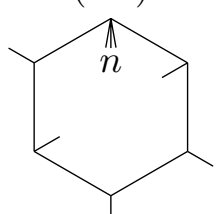

(6.5)

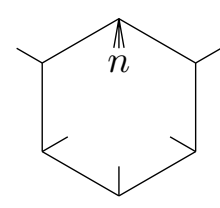

(6.2)

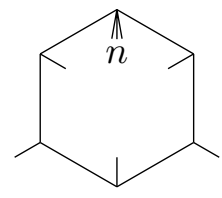

(6.4)

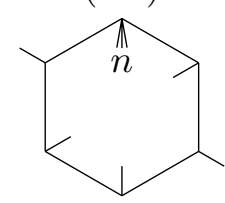

(6.6)

Figure 30: Configurations from the identifications among $x_{*}, y_{*}, z_{*}$.

only need to consider the generic reductions in Figure 31. A subtle point in constructing the reductions is to make sure that all the newly created vertices are of distance $\leqslant 3$ from the high degree boundary vertex, so that the new vertices have degree 3 .
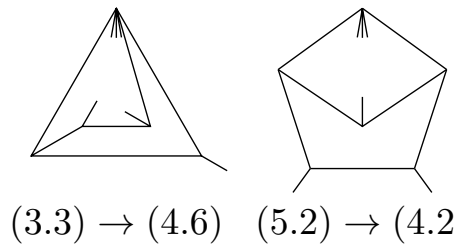

$(5.2) \rightarrow(4.2)$
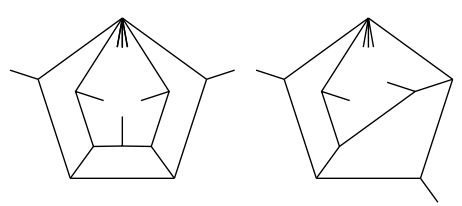

$(5.1) \rightarrow(6.4)$

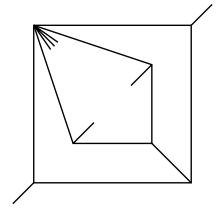

$(4.2) \rightarrow(4.3)$

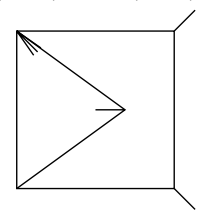

$(4.5) \rightarrow(3.3)$

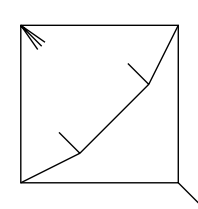

$(4.3) \rightarrow(5.1)$

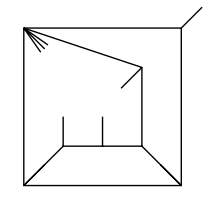

$(4.6) \rightarrow(6.6)$
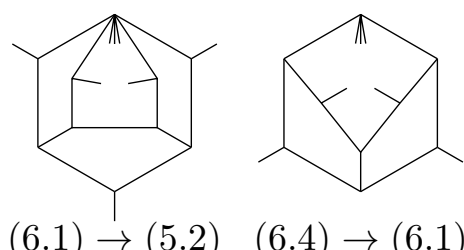

$(6.1) \rightarrow(5.2)$

$(6.4) \rightarrow(6.1)$
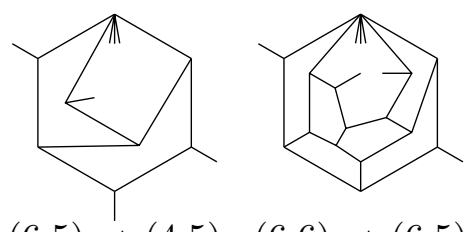

$(6.5) \rightarrow(4.5)$

Figure 31: Generic reductions of configurations in Figure 30.

We also need to consider the configurations with small $n$ so that the generic reductions cannot be carried out. By Figure 28, the configurations $(3.3)_{n=0},(4.2)_{n=0},(4.5)_{n=0}$ are impossible. The reductions of the remaining special configurations are given by Figure 32. The question mark means that the configuration appears in Figure 28. 

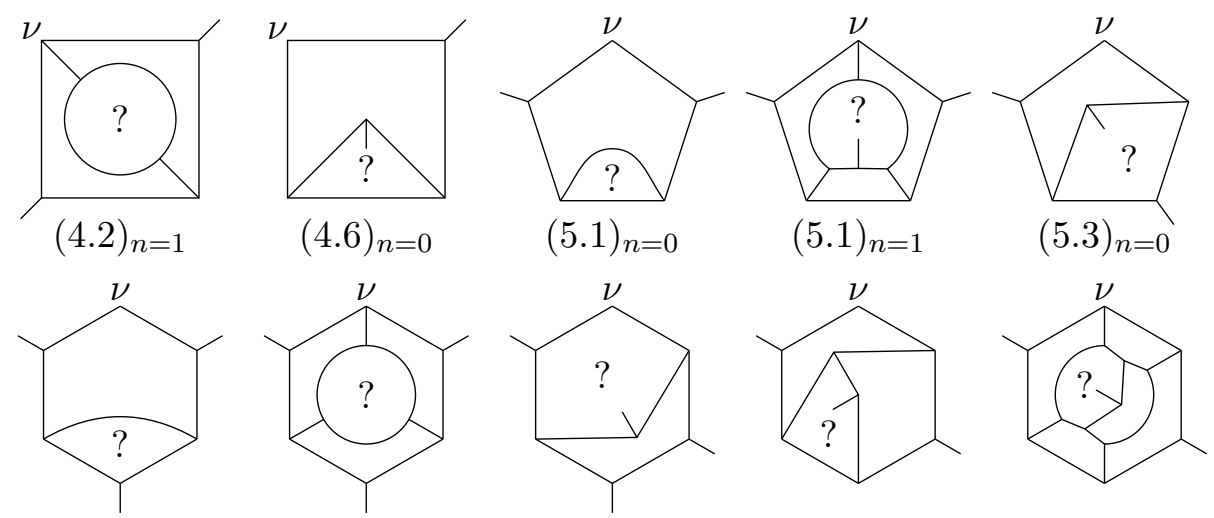

$(6.1)_{n=0}$

$(6.1)_{n=1}$

$(6.5)_{n=0}$

$(6.6)_{n=0}$

$(6.6)_{n=0}$

Figure 32: Special reductions of configurations in Figure 30.

After proving that $x_{*}, y_{*}, z_{*}$ are all distinct, we get two layers of tiles around $\nu$ as expected. Then we need to further construct another layer of tiles with $z_{*}$ and $w_{*}$ as vertices. For this part, the original argument is still correct. 